\title{
Effect of Nitrogen and Spacing Levels on Physiological and Yield Parameters of Kasuri Methi (Trigonella corniculata L.) var. Pusa Kasuri
}

\author{
G. Anupama*, L.N. Hegde, N.K. Hegde, V. Devappa, A.B. Mastiholi and \\ Sandhyarani Nishani
}

Department of Plantation, Spices, Medicinal and Aromatic plants, Kittur Rani Channamma College of Horticulture, Arabhavi-591 310,University of Horticultural Sciences, Bagalkot- 587 102, Karnataka, India

*Corresponding author

\section{A B S T R A C T}

\section{Keywords \\ Kasuri methi (Trigonella corniculata $\mathrm{L}$.) var. Pusa Kasuri, Nitrogen levels, Spacing levels, \\ Physiological and yield parameters. \\ Article Info \\ Accepted: \\ 14 July 2017 \\ Available Online: \\ 10 September 2017}

A field experiment was conducted with 4 nitrogen and 4 spacing levels in factorial randomized block design with three replications. Maximum leaf area, chlorophyll content, AGR and fresh and dry yield per plant were recorded when the crop was applied with $80 \mathrm{~kg}$ N/ha with a spacing of $20 \mathrm{x}$ $10 \mathrm{~cm}\left(\mathrm{~N}_{4} \mathrm{~S}_{1}\right)$ whereas the plant spaced at $20 \times 10 \mathrm{~cm}\left(\mathrm{~S}_{1}\right)$ recorded maximum CGR, LAI, fresh and dry yield per hectare under the Northern dry zone of Karnataka.

\section{Introduction}

Kasuri methi (Trigonella corniculata L.) is a semi-arid crop belonging to the family Fabaceae. It is commonly known as Champa methi' and 'marwari methi', is a diffused sub erects and strongly scented annual herb. It remains rosette condition during most of the vegetative growth period (Anon., 2004). It is originated in Mediterranean region and near east countries. The dried leaves are the economic part of this plant. In India, it is mainly grown in northern states like Maharashtra Rajasthan, Gujarat, Uttarpradesh, Madhya Pradesh, Haryana and Punjab. Rajasthan occupies 80 per cent area and production of Kasuri methi in the country.

Kasuri methi is a multipurpose crop. Its every part is useful and is utilized in one or other forms as food, fodder, medicine and cosmetics. The fresh green leaves are used as condiment for giving delicious flavor.

These have an aromatic odor and agreeable spicy taste. Being odoriferous, the dried seeds and their powder are used as condiment flavoring agent and for medicinal purposes. Kasuri methi has many uses such as flavoring 
and medicinal purpose, is gaining importance in recent years and there is a good demand for the dried leaves in the market. The dried leaves are regularly sold in the super markets as popular flavoring material. The scientific information on cultivation and nutrient management of this crop is very less. Hence, present investigation is taken up to study its physiological and yield performance in southern part of India, particularly in Northern dry zone of Karnataka.

Considering the above facts, the present investigation was undertaken with the following objectives to study effect of nitrogen, spacing levels and their interaction on physiological and yield parameters of Kasuri methi var. Pusa Kasuri.

\section{Materials and Methods}

A field experiment was conducted at the department of Plantation, Spices, Medicinal and Aromatic Crops, Kittur Rani Channamma College of Horticulture, Arabhavi, University of Horticultural Sciences, Bagalkot from November, 2011 to January, 2012. Geographically, the Arabhavi lies in the Zone-3 of Region-2 in the agro-climatic zone of Karnataka. It is situated at $16^{\mathrm{O}} 15^{\prime}$ North latitude and $74^{\circ} 45^{\prime}$ East longitude and at an altitude of $612 \mathrm{~m}$ above the mean sea level. The soil of the experimental site was medium deep black in texture. The experiment was laid out with 4 levels of nitrogen $\mathrm{N}_{1}-20 \mathrm{~kg}$, $\mathrm{N}_{2}-40 \mathrm{~kg}, \mathrm{~N}_{3}-60 \mathrm{~kg}$ and $\mathrm{N}_{4}-80 \mathrm{~kg} \mathrm{~N} / \mathrm{ha}$ and spacing levels viz., $S_{1^{-}} 20 \times 10 \mathrm{~cm}, S_{2^{-}} 20 \times$ $20 \mathrm{~cm}, S_{3^{-}} 30 \times 10 \mathrm{~cm}$ and $S_{4^{-}} 30 \times 20 \mathrm{~cm}$. It was laid out in RBD with factorial concept in three replications.

Healthy seeds of variety Pusa Kasuri were used for sowing. They were sown on $28^{\text {th }}$ November, 2011 as per the s above mentioned spacings. Furrows were properly covered with a thin layer of soil and the plots were irrigated with small stream of water. Farm yard manure was applied one week before the sowing of crop at the rate of 10 tons per hectare as a common dose for all the treatments. The plots were supplied with half the dose of nitrogen (in the form of urea) and full dose of phosphorus (in the form of single super phosphate) as per the treatments. The remaining half dose of nitrogen was supplemented 30 days after sowing as top dressing and the light earthing up was done. Observations on growth and yield parameters were recorded using five plants per plot and the data collected during the study was subjected to Statistical analysis using the Fischer's method of analysis of variance technique as given by Panse and Sukhatme (1967).

\section{Results and Discussion}

\section{Physiological parameters}

\section{Leaf area and leaf area index}

The observations with respect to the effect of nitrogen, spacing and their interaction on leaf area at harvest and leaf area index are presented in table 1.

Among the nitrogen levels, $\mathrm{N}_{4}$ recorded significantly higher leaf area $\left(1683.32 \mathrm{~cm}^{2}\right)$. Whereas, the minimum leaf area $\left(344.51 \mathrm{~cm}^{2}\right)$ was noticed in the $\mathrm{N}_{1}$. Among the spacing level, the plants spaced at $30 \times 20 \mathrm{~cm}\left(\mathrm{~S}_{4}\right)$ recorded significantly higher leaf area $\left(1109.96 \mathrm{~cm}^{2}\right)$, whereas, the minimum leaf area $\left(745.66 \mathrm{~cm}^{2}\right)$ was noticed in $\mathrm{S}_{1}(20 \mathrm{x} 10$ $\mathrm{cm})$. The significant variation in leaf area at harvest is due to interaction of nitrogen and spacing levels. However, the maximum $\mathrm{N}_{4} \mathrm{~S}_{4}$ (1964.21 $\left.\mathrm{cm}^{2}\right)$, whereas, the minimum $\left(220.14 \mathrm{~cm}^{2}\right)$ leaf area recorded in $\mathrm{N}_{1} \mathrm{~S}_{1}$.

Among the nitrogen levels, $\mathrm{N}_{4}$ recorded significantly higher leaf area index (4.40). 
While, the minimum leaf area index (0.98) was noticed in the $\mathrm{N}_{1}$. Among the spacing levels, the plants spaced at $20 \times 10 \mathrm{~cm}\left(\mathrm{~S}_{1}\right)$ recorded significantly higher leaf area index (3.73). Whereas the minimum leaf area index (1.85) was noticed in $\mathrm{S}_{4}(30 \times 20 \mathrm{~cm})$. The significant variation in leaf area index at harvest due to interaction of nitrogen and spacing levels. However, the maximum LAI observed in treatment combination of $\mathrm{N}_{4} \mathrm{~S}_{1}$ (7.56). Whereas, the minimum (0.74) LAI observed in $\mathrm{N}_{1} \mathrm{~S}_{4}$.

Linear increases in leaf area and leaf area index were observed with increase in the nitrogen levels. This could be due to production of more number of leaves, branches and enhanced availability of nitrogen at the appropriate time, which has increased the leaf area and LAI. These results are in accordance with the findings of Mehta et al., (2010) in fenugreek, and Kattimani (1999) in Mentha arvensis L.

\section{Absolute Growth Rate (AGR)}

The observations with respect to the effect of different levels of nitrogen, spacing and their interaction on absolute growth rate (AGR) are presented in table 2. At 30-45 Days after sowing, the application of nitrogen recorded higher AGR in $\mathrm{N}_{4}(0.274 \mathrm{~g} /$ day $)$ and least AGR (0.162g/day) in $\mathrm{N}_{1}$ whereas plant density at $\mathrm{S}_{4}$ recorded maximum AGR (0.240 $\mathrm{g}$ /day) and the minimum AGR observed in $\mathrm{S}_{1}$ $(0.204 \mathrm{~g} /$ day $)$. The interaction between nitrogen and plant densities differs significantly for AGR. The maximum AGR was observed in treatment combination of $\mathrm{N}_{4} \mathrm{~S}_{4}(0.283 \mathrm{~g} /$ day $)$ followed by, $\mathrm{N}_{4} \mathrm{~S}_{3}(0.278$ g/day), $\mathrm{N}_{4} \mathrm{~S}_{2}(0.272 \mathrm{~g} /$ day $)$ and $\mathrm{N}_{4} \mathrm{~S}_{1}(0.264$ g/day).While, the minimum AGR was recorded in $\mathrm{N}_{1} \mathrm{~S}_{1}$ (0.135 g/day). At 46-60 DAS the Maximum AGR recorded in $\mathrm{N}_{4}$ (0.295 g/day) whereas minimum AGR (0.214 $\mathrm{g}$ /day) was noticed in $\mathrm{N}_{1}$. The plants spaced at
$30 \times 20 \mathrm{~cm}\left(\mathrm{~S}_{4}\right)$ recorded the maximum AGR $(0.257 \mathrm{~g} /$ day $)$ followed by $\mathrm{S}_{3}(0.247 \mathrm{~g} /$ day $)$ while the minimum was recorded in $\mathrm{S}_{1}(0.227$ $\mathrm{g} /$ day). The interaction between nitrogen and plant densities differed significantly for AGR. The higher AGR was observed in $\mathrm{N}_{4} \mathrm{~S}_{4}(0.322$ g/day) followed by, $\mathrm{N}_{4} \mathrm{~S}_{3} \quad(0.310$ $\mathrm{g} /$ day). While, the least AGR was recorded in $\mathrm{N}_{1} \mathrm{~S}_{1}$ (0.204 g/day). At 61-75 Days after sowing, the maximum AGR recorded in $80 \mathrm{~kg}$ $\mathrm{N}$ per ha $\left(\mathrm{N}_{4}\right)(0.431 \mathrm{~g} /$ day $)$.

Whereas the minimum AGR (0.262 g/day) was recorded in $\mathrm{N}_{1}$. Whereas plant density at $\mathrm{S}_{4}(0.382 \mathrm{~g} /$ day $)$ recorded maximum AGR followed by, $S_{3}(0.365 \mathrm{~g} /$ day $)$. While the minimum AGR observed in $\mathrm{S}_{1}(0.337 \mathrm{~g} /$ day). The interactions between nitrogen and plant densities differ significantly for AGR. The maximum AGR were observed in $\mathrm{N}_{4} \mathrm{~S}_{4}(0.445$ $\mathrm{g}$ /day) followed by, $\mathrm{N}_{4} \mathrm{~S}_{3}(0.432 \mathrm{~g} /$ day $), \mathrm{N}_{4} \mathrm{~S}_{2}$ (0.430 g/day), $\mathrm{N}_{4} \mathrm{~S}_{1}(0.417 \mathrm{~g} /$ day $)$ and $\mathrm{N}_{3} \mathrm{~S}_{4}$ $(0.411 \mathrm{~g} /$ day $)$. While the minimum AGR were observed in $\mathrm{N}_{1} \mathrm{~S}_{1}(0.238 \mathrm{~g} /$ day $)$.

The absolute growth rate was significant at all the stages of crop growth. This may be due to better vegetative growth in terms of highest plant height, number of leaves, branches and total dry matter accumulation in the plants supplied with higher levels of nitrogen. Parallel trend was observed by Patidar et al., (2004) in cumin.

\section{Cumulative Growth Rate (CGR)}

The observations with respect to the effect of different levels of nitrogen, spacing levels and their interaction on cumulative growth rate (CGR) are presented in table 3. At 30-45 Days after sowing, the application of nitrogen recorded higher CGR in $\mathrm{N}_{4}(8.50 \mathrm{~g} / \mathrm{m} 2 /$ day $)$ and least (4.84 g/m2/day) was in $\mathrm{N}_{1}$ whereas plant spaced at $S_{1}$ recorded maximum (10.18 $\mathrm{g} / \mathrm{m} 2 /$ day) CGR while the minimum CGR was observed in $\mathrm{S}_{4}\left(3.99 \mathrm{~g} / \mathrm{m}^{2} /\right.$ day $)$. 
Table.1 Effect of nitrogen and spacing levels on leaf area and leaf area Index (at 60 DAS) of kasuri methi (Trigonella corniculata L.) var. Pusa Kasuri

\begin{tabular}{|c|c|c|}
\hline Treatment & Leaf area $\left(\mathrm{cm}^{2}\right)$ & Leaf area Index \\
\hline \multicolumn{3}{|l|}{ Nitrogen level (N) } \\
\hline$N_{1}: 20 \mathrm{~kg} / \mathrm{ha}$ & 344.51 & 0.98 \\
\hline $\mathrm{N}_{2}: 40 \mathrm{~kg} / \mathrm{ha}$ & 568.88 & 1.70 \\
\hline $\mathrm{N}_{3}: 60 \mathrm{~kg} / \mathrm{ha}$ & 1062.31 & 3.10 \\
\hline $\mathrm{N}_{4}: 80 \mathrm{~kg} / \mathrm{ha}$ & 1683.32 & 4.40 \\
\hline $\operatorname{Mean}(\mathbf{N})$ & 914.75 & 2.54 \\
\hline S.Em \pm & 22.24 & 0.06 \\
\hline CD@ $5 \%$ & 64.25 & 0.18 \\
\hline \multicolumn{3}{|l|}{ Spacing level (S) } \\
\hline$S_{1}: 20 \times 10 \mathrm{~cm}(50,000$ plants $/ \mathrm{ha})$ & 745.66 & 3.73 \\
\hline$S_{2}: 20 \times 20 \mathrm{~cm}(25,000$ plants/ha $)$ & 855.70 & 2.14 \\
\hline$S_{3}: 30 \times 10 \mathrm{~cm}(3,33000$ plants $/ \mathrm{ha})$ & 947.69 & 2.46 \\
\hline$S_{4}: 30 \times 20 \mathrm{~cm}(1,66000$ plants $/ \mathrm{ha})$ & 1109.96 & 1.85 \\
\hline Mean (S) & 914.75 & 2.54 \\
\hline S.Em \pm & 22.24 & 0.06 \\
\hline CD@ $5 \%$ & 64.25 & 0.18 \\
\hline \multicolumn{3}{|l|}{ Interaction $(\mathrm{N} \times \mathrm{S})$} \\
\hline $\mathbf{N}_{1} \mathbf{S}_{1}$ & 220.14 & 1.10 \\
\hline $\mathbf{N}_{1} \mathbf{S}_{2}$ & 342.34 & 0.86 \\
\hline $\mathbf{N}_{1} \mathbf{S}_{3}$ & 373.15 & 1.24 \\
\hline $\mathbf{N}_{1} \mathbf{S}_{4}$ & 442.40 & 0.74 \\
\hline $\mathbf{N}_{2} \mathbf{S}_{1}$ & 460.12 & 2.30 \\
\hline $\mathbf{N}_{2} \mathbf{S}_{2}$ & 578.71 & 1.45 \\
\hline $\mathbf{N}_{2} \mathbf{S}_{3}$ & 591.10 & 1.97 \\
\hline $\mathbf{N}_{2} \mathbf{S}_{4}$ & 645.59 & 1.08 \\
\hline $\mathbf{N}_{3} \mathbf{S}_{1}$ & 790.99 & 3.95 \\
\hline $\mathbf{N}_{3} \mathbf{S}_{2}$ & 917.73 & 2.29 \\
\hline $\mathbf{N}_{3} \mathbf{S}_{3}$ & 1152.86 & 3.84 \\
\hline $\mathbf{N}_{3} \mathbf{S}_{4}$ & 1387.66 & 2.31 \\
\hline $\mathbf{N}_{4} \mathbf{S}_{1}$ & 1511.40 & 7.56 \\
\hline $\mathbf{N}_{4} \mathbf{S}_{2}$ & 1584.01 & 3.96 \\
\hline $\mathbf{N}_{4} \mathbf{S}_{3}$ & 1673.66 & 2.79 \\
\hline $\mathbf{N}_{4} \mathbf{S}_{4}$ & 1964.21 & 3.27 \\
\hline Mean $(\mathrm{N} \times \mathrm{S})$ & 914.75 & 2.54 \\
\hline S.Em \pm & 44.49 & 0.13 \\
\hline CD@ 5 \% & 128.49 & 0.36 \\
\hline
\end{tabular}


Table.2 Effect of nitrogen and spacing levels on AGR (absolute growth rate) of Kasuri methi (Trigonella corniculata L.) var. Pusa Kasuri

\begin{tabular}{|c|c|c|c|}
\hline \multirow{2}{*}{ Treatment } & \multicolumn{3}{|c|}{$\begin{array}{l}\text { Absolute growth rate (g/day) } \\
\text { at different stages of plant growth }\end{array}$} \\
\hline & 30-45 DAS & 46-60 DAS & 61 -75 DAS \\
\hline \multicolumn{4}{|l|}{ Nitrogen level (N) } \\
\hline$N_{1}: 20 \mathrm{~kg} / \mathrm{ha}$ & 0.162 & 0.214 & 0.262 \\
\hline $\mathrm{N}_{2}: 40 \mathrm{~kg} / \mathrm{ha}$ & 0.210 & 0.225 & 0.342 \\
\hline$N_{3}: 60 \mathrm{~kg} / \mathrm{ha}$ & 0.241 & 0.230 & 0.402 \\
\hline$N_{4}: 80 \mathrm{~kg} / \mathrm{ha}$ & 0.274 & 0.295 & 0.431 \\
\hline Mean $(\mathbf{N})$ & 0.222 & 0.241 & 0.359 \\
\hline S.Em \pm & 0.004 & 0.006 & 0.006 \\
\hline CD@ $15 \%$ & 0.011 & 0.018 & 0.017 \\
\hline \multicolumn{4}{|l|}{ Spacing level (S) } \\
\hline$S_{1}: 20 \times 10 \mathrm{~cm}(50,000$ plants $/ \mathrm{ha})$ & 0.204 & 0.227 & 0.337 \\
\hline$S_{2}: 20 \times 20 \mathrm{~cm}(25,000$ plants/ha $)$ & 0.217 & 0.233 & 0.353 \\
\hline$S_{3}: 30 \times 10 \mathrm{~cm}(3,33000$ plants/ha $)$ & 0.227 & 0.247 & 0.365 \\
\hline$S_{4}: 30 \times 20 \mathrm{~cm}(1,66000$ plants $/ \mathrm{ha})$ & 0.240 & 0.257 & 0.382 \\
\hline Mean (S) & 0.240 & 0.241 & 0.359 \\
\hline S.Em \pm & 0.222 & 0.006 & 0.006 \\
\hline CD @ $5 \%$ & 0.011 & 0.018 & 0.017 \\
\hline \multicolumn{4}{|l|}{ Interaction ( $\mathbf{N}$ x S) } \\
\hline $\mathbf{N}_{1} \mathbf{S}_{1}$ & 0.135 & 0.204 & 0.238 \\
\hline $\mathbf{N}_{1} \mathbf{S}_{2}$ & 0.151 & 0.212 & 0.254 \\
\hline $\mathbf{N}_{1} \mathbf{S}_{3}$ & 0.169 & 0.216 & 0.273 \\
\hline $\mathbf{N}_{1} \mathbf{S}_{4}$ & 0.193 & 0.223 & 0.282 \\
\hline $\mathbf{N}_{2} \mathbf{S}_{1}$ & 0.187 & 0.214 & 0.302 \\
\hline $\mathbf{N}_{2} \mathbf{S}_{2}$ & 0.210 & 0.215 & 0.327 \\
\hline $\mathbf{N}_{2} \mathbf{S}_{3}$ & 0.214 & 0.233 & 0.349 \\
\hline $\mathbf{N}_{2} \mathbf{S}_{4}$ & 0.230 & 0.239 & 0.388 \\
\hline $\mathbf{N}_{3} \mathbf{S}_{1}$ & 0.229 & 0.220 & 0.392 \\
\hline $\mathbf{N}_{3} \mathbf{S}_{2}$ & 0.237 & 0.228 & 0.401 \\
\hline $\mathbf{N}_{3} \mathbf{S}_{3}$ & 0.247 & 0.229 & 0.405 \\
\hline $\mathbf{N}_{3} \mathbf{S}_{4}$ & 0.252 & 0.244 & 0.411 \\
\hline $\mathbf{N}_{4} \mathbf{S}_{1}$ & 0.264 & 0.270 & 0.417 \\
\hline $\mathbf{N}_{4} \mathbf{S}_{2}$ & 0.272 & 0.278 & 0.430 \\
\hline $\mathbf{N}_{4} \mathbf{S}_{3}$ & 0.278 & 0.310 & 0.432 \\
\hline $\mathbf{N}_{4} \mathbf{S}_{4}$ & 0.283 & 0.322 & 0.445 \\
\hline Mean $(\mathrm{N} x \mathrm{~S})$ & 0.222 & 0.241 & 0.359 \\
\hline S.Em \pm & 0.007 & 0.012 & 0.012 \\
\hline CD@ $5 \%$ & 0.021 & 0.035 & 0.034 \\
\hline
\end{tabular}

* DAS- Days after sowing 
Table.3 Effect of nitrogen and spacing levels on CGR (cumulative growth rate) of Kasuri methi (Trigonella corniculata L.) var. Pusa Kasuri

\begin{tabular}{|c|c|c|c|}
\hline \multirow[b]{2}{*}{ Treatment } & \multicolumn{3}{|c|}{$\begin{array}{l}\text { Cumulative Growth Rate }\left(\mathrm{g} / \mathrm{m}^{2} / \mathrm{day}\right) \\
\text { at different stage of plant growth }\end{array}$} \\
\hline & 30-45 DAS & 46-60 DAS & 61-75 DAS \\
\hline \multicolumn{4}{|l|}{ Nitrogen level (N) } \\
\hline$N_{1}: 20 \mathrm{~kg} / \mathrm{ha}$ & 4.84 & 6.61 & 8.02 \\
\hline$N_{2}: 40 \mathrm{~kg} / \mathrm{ha}$ & 6.39 & 6.95 & 10.34 \\
\hline$N_{3}: 60 \mathrm{~kg} / \mathrm{ha}$ & 7.45 & 7.10 & 12.48 \\
\hline$N_{4}: 80 \mathrm{~kg} / \mathrm{ha}$ & 8.50 & 9.04 & 13.34 \\
\hline $\operatorname{Mean}(\mathbf{N})$ & 6.80 & 7.42 & 11.05 \\
\hline S.Em \pm & 0.11 & 0.24 & 0.25 \\
\hline CD@ $5 \%$ & 0.32 & 0.70 & 0.72 \\
\hline \multicolumn{4}{|l|}{ Spacing level (S) } \\
\hline$S_{1}: 20 \times 10 \mathrm{~cm}(50,000$ plants/ha $)$ & 10.18 & 11.34 & 16.85 \\
\hline$S_{2}: 20 \times 20 \mathrm{~cm}(25,000$ plants/ha $)$ & 5.44 & 5.83 & 8.82 \\
\hline$S_{3}: 30 \times 10 \mathrm{~cm}(3,33000$ plants $/ \mathrm{ha})$ & 7.57 & 8.24 & 12.15 \\
\hline$S_{4}: 30 \times 20 \mathrm{~cm}(1,66000$ plants $/ \mathrm{ha})$ & 3.99 & 4.28 & 6.36 \\
\hline Mean (S) & 6.80 & 87.42 & 11.05 \\
\hline S.Em \pm & 0.11 & 0.24 & 0.25 \\
\hline CD@ $5 \%$ & 0.32 & 0.70 & 0.72 \\
\hline \multicolumn{4}{|l|}{ Interaction $(\mathrm{N} \times \mathrm{S})$} \\
\hline $\mathbf{N}_{1} \mathbf{S}_{1}$ & 6.74 & 10.20 & 11.90 \\
\hline $\mathbf{N}_{1} \mathbf{S}_{2}$ & 3.77 & 5.31 & 6.36 \\
\hline $\mathbf{N}_{1} \mathbf{S}_{3}$ & 5.64 & 7.21 & 9.10 \\
\hline $\mathbf{N}_{1} \mathbf{S}_{4}$ & 3.22 & 3.72 & 4.71 \\
\hline $\mathbf{N}_{2} \mathbf{S}_{1}$ & 9.34 & 10.68 & 15.10 \\
\hline $\mathbf{N}_{2} \mathbf{S}_{2}$ & 5.24 & 5.37 & 8.18 \\
\hline $\mathbf{N}_{2} \mathbf{S}_{3}$ & 7.13 & 7.77 & 11.62 \\
\hline $\mathbf{N}_{2} \mathbf{S}_{4}$ & 3.84 & 3.99 & 6.47 \\
\hline $\mathbf{N}_{3} \mathbf{S}_{1}$ & 11.43 & 11.00 & 19.58 \\
\hline $\mathbf{N}_{3} \mathbf{S}_{2}$ & 5.93 & 5.71 & 10.02 \\
\hline $\mathbf{N}_{3} \mathbf{S}_{3}$ & 8.24 & 7.64 & 13.50 \\
\hline $\mathbf{N}_{3} \mathbf{S}_{4}$ & 4.20 & 4.06 & 6.85 \\
\hline $\mathbf{N}_{4} \mathbf{S}_{1}$ & 13.21 & 13.49 & 20.83 \\
\hline $\mathbf{N}_{4} \mathbf{S}_{2}$ & 6.80 & 6.96 & 10.74 \\
\hline $\mathbf{N}_{4} \mathbf{S}_{3}$ & 9.26 & 10.34 & 14.39 \\
\hline $\mathbf{N}_{4} \mathbf{S}_{4}$ & 4.71 & 5.36 & 7.41 \\
\hline Mean (N x S) & 6.80 & 7.42 & 11.05 \\
\hline S.Em \pm & 0.22 & 0.48 & 0.50 \\
\hline CD@ $5 \%$ & 0.64 & 0.39 & 1.43 \\
\hline
\end{tabular}

* DAS- Days after sowing 
Table.4 Effect of nitrogen and spacing levels on chlorophyll contents in kasuri methi (Trigonella corniculata L.) var. Pusa Kasuri

\begin{tabular}{|c|c|c|c|}
\hline \multirow[b]{2}{*}{ Treatment } & \multicolumn{3}{|c|}{ Chlorophyll contents $(\mathrm{mg} / 100 \mathrm{mg}$ of tissue at $60 \mathrm{DAS})$} \\
\hline & $\begin{array}{c}\text { Chlorophyll- } \\
\text { 'a' }\end{array}$ & $\begin{array}{c}\text { Chlorophyll- } \\
\text { 'b' }\end{array}$ & $\begin{array}{c}\text { Total chlorophyll } \\
\text { content }\end{array}$ \\
\hline \multicolumn{4}{|l|}{ Nitrogen level $(\mathbf{N})$} \\
\hline$N_{1}: 20 \mathrm{~kg} / \mathrm{ha}$ & 0.78 & 0.30 & 1.08 \\
\hline $\mathrm{N}_{2}: 40 \mathrm{~kg} / \mathrm{ha}$ & 1.41 & 0.36 & 1.77 \\
\hline$N_{3}: 60 \mathrm{~kg} / \mathrm{ha}$ & 1.50 & 0.76 & 2.26 \\
\hline $\mathrm{N}_{4}: 80 \mathrm{~kg} / \mathrm{ha}$ & 1.61 & 1.38 & 2.99 \\
\hline $\operatorname{Mean}(\mathbf{N})$ & 1.32 & 0.70 & 2.02 \\
\hline S.Em \pm & 0.02 & 0.05 & 0.05 \\
\hline CD@ $5 \%$ & 0.07 & 0.14 & 0.14 \\
\hline \multicolumn{4}{|l|}{ Spacing level (S) } \\
\hline$S_{1}: 20 \times 10 \mathrm{~cm}(50,000$ plants $/ \mathrm{ha})$ & 1.00 & 0.48 & 1.47 \\
\hline$S_{2}: 20 \times 20 \mathrm{~cm}(25,000$ plants/ha $)$ & 1.29 & 0.59 & 1.88 \\
\hline$S_{3}: 30 \times 10 \mathrm{~cm}(3,33000$ plants $/ \mathrm{ha})$ & 1.46 & 0.77 & 2.23 \\
\hline $\mathrm{S}_{4}: 30 \times 20 \mathrm{~cm}(1,66000$ plants $/ \mathrm{ha})$ & 1.55 & 0.96 & 2.51 \\
\hline Mean (S) & 1.32 & 0.70 & 2.02 \\
\hline S.Em \pm & 0.02 & 0.05 & 0.05 \\
\hline CD@ $5 \%$ & 0.07 & 0.14 & 0.14 \\
\hline \multicolumn{4}{|l|}{ Interaction (N x S) } \\
\hline $\mathbf{N}_{1} \mathbf{S}_{1}$ & 0.14 & 0.04 & 0.17 \\
\hline $\mathbf{N}_{1} \mathbf{S}_{2}$ & 0.73 & 0.23 & 0.96 \\
\hline $\mathbf{N}_{1} \mathbf{S}_{3}$ & 1.12 & 0.33 & 1.45 \\
\hline $\mathbf{N}_{1} \mathbf{S}_{4}$ & 1.13 & 0.59 & 1.72 \\
\hline $\mathbf{N}_{2} \mathbf{S}_{1}$ & 1.29 & 0.24 & 1.52 \\
\hline $\mathbf{N}_{2} \mathbf{S}_{2}$ & 1.37 & 0.27 & 1.63 \\
\hline $\mathbf{N}_{2} \mathbf{S}_{3}$ & 1.37 & 0.43 & 1.80 \\
\hline $\mathbf{N}_{2} \mathbf{S}_{4}$ & 1.60 & 0.51 & 2.11 \\
\hline $\mathbf{N}_{3} \mathbf{S}_{1}$ & 1.35 & 0.56 & 1.91 \\
\hline $\mathbf{N}_{3} \mathbf{S}_{2}$ & 1.36 & 0.65 & 2.00 \\
\hline $\mathbf{N}_{3} \mathbf{S}_{3}$ & 1.63 & 0.78 & 2.41 \\
\hline $\mathbf{N}_{3} \mathbf{S}_{4}$ & 1.65 & 1.06 & 2.74 \\
\hline $\mathbf{N}_{4} \mathbf{S}_{1}$ & 1.22 & 1.07 & 2.29 \\
\hline $\mathbf{N}_{4} \mathbf{S}_{2}$ & 1.71 & 1.21 & 2.93 \\
\hline $\mathbf{N}_{4} \mathbf{S}_{3}$ & 1.72 & 1.55 & 3.26 \\
\hline $\mathbf{N}_{4} \mathbf{S}_{4}$ & 1.80 & 1.67 & 3.48 \\
\hline Mean $(\mathrm{N} x \mathrm{~S})$ & 1.32 & 0.70 & 2.02 \\
\hline S.Em \pm & 0.05 & 0.10 & 0.09 \\
\hline CD@ $5 \%$ & 0.14 & 0.28 & 0.27 \\
\hline
\end{tabular}


Table.5 Effect of nitrogen and spacing levels on fresh and dry yield of Kasuri methi (Trigonella corniculata L.) var. Pusa Kasuri

\begin{tabular}{|c|c|c|c|c|}
\hline Treatment & $\begin{array}{c}\text { Fresh } \\
\text { yield } \\
\text { (g/plant) }\end{array}$ & $\begin{array}{c}\text { Fresh yield } \\
\text { (t/ha) }\end{array}$ & $\begin{array}{c}\text { Dry yield } \\
\text { (g/plant) }\end{array}$ & $\begin{array}{c}\text { Dry yield } \\
\text { (t/ha) }\end{array}$ \\
\hline \multicolumn{5}{|l|}{ Nitrogen level (N) } \\
\hline $\mathrm{N}_{1}: 20 \mathrm{~kg} / \mathrm{ha}$ & 55.23 & 17.15 & 11.00 & 3.42 \\
\hline $\mathrm{N}_{2}: 40 \mathrm{~kg} / \mathrm{ha}$ & 57.87 & 17.99 & 11.58 & 3.59 \\
\hline$N_{3}: 60 \mathrm{~kg} / \mathrm{ha}$ & 59.61 & 18.59 & 12.10 & 3.77 \\
\hline$N_{4}: 80 \mathrm{~kg} / \mathrm{ha}$ & 63.36 & 19.61 & 12.55 & 3.90 \\
\hline Mean $(\mathbf{N})$ & 59.02 & 18.33 & 11.81 & 3.67 \\
\hline S.Em \pm & 0.42 & 0.13 & 0.03 & 0.01 \\
\hline CD@ $5 \%$ & 1.22 & 0.39 & 0.08 & 0.03 \\
\hline \multicolumn{5}{|l|}{ Spacing level (S) } \\
\hline$S_{1}: 20 \times 10 \mathrm{~cm}(50,000$ plants/ha $)$ & 57.77 & 28.88 & 11.60 & 5.80 \\
\hline$S_{2}: 20 \times 20 \mathrm{~cm}(25,000$ plants/ha $)$ & 58.4 & 14.6 & 11.72 & 2.93 \\
\hline$S_{3}: 30 \times 10 \mathrm{~cm}(3,33000$ plants $/ \mathrm{ha})$ & 59.54 & 19.83 & 11.87 & 3.95 \\
\hline $\mathrm{S}_{4}: 30 \times 20 \mathrm{~cm}(1,66000$ plants $/ \mathrm{ha})$ & 60.36 & 10.02 & 12.04 & 2.00 \\
\hline Mean (S) & 59.02 & 18.33 & 11.81 & 3.67 \\
\hline S.Em \pm & 0.42 & 0.13 & 0.03 & 0.01 \\
\hline CD@ $5 \%$ & 1.22 & 0.39 & 0.08 & 0.03 \\
\hline \multicolumn{5}{|l|}{ Interaction $(\mathbf{N} \times \mathbf{S})$} \\
\hline $\mathbf{N}_{1} \mathbf{S}_{1}$ & 54.02 & 27.01 & 10.74 & 5.37 \\
\hline $\mathbf{N}_{1} \mathbf{S}_{2}$ & 54.55 & 13.64 & 10.90 & 2.73 \\
\hline $\mathbf{N}_{1} \mathbf{S}_{3}$ & 55.7 & 18.55 & 11.11 & 3.70 \\
\hline $\mathbf{N}_{1} \mathbf{S}_{4}$ & 56.63 & 9.4 & 11.25 & 1.87 \\
\hline $\mathbf{N}_{2} \mathbf{S}_{1}$ & 56.63 & 28.32 & 11.29 & 5.65 \\
\hline $\mathbf{N}_{2} \mathbf{S}_{2}$ & 57.48 & 14.37 & 11.46 & 2.86 \\
\hline $\mathbf{N}_{2} \mathbf{S}_{3}$ & 58.57 & 19.5 & 11.67 & 3.89 \\
\hline $\mathbf{N}_{2} \mathbf{S}_{4}$ & 58.8 & 9.76 & 11.88 & 1.97 \\
\hline $\mathbf{N}_{3} \mathbf{S}_{1}$ & 59.22 & 29.61 & 11.97 & 5.99 \\
\hline $\mathbf{N}_{3} \mathbf{S}_{2}$ & 59.59 & 14.9 & 12.07 & 3.02 \\
\hline $\mathbf{N}_{3} \mathbf{S}_{3}$ & 59.73 & 19.89 & 12.13 & 4.04 \\
\hline $\mathbf{N}_{3} \mathbf{S}_{4}$ & 59.9 & 9.94 & 12.24 & 2.03 \\
\hline $\mathbf{N}_{4} \mathbf{S}_{1}$ & 61.2 & 30.6 & 12.38 & 6.19 \\
\hline $\mathbf{N}_{4} \mathbf{S}_{2}$ & 61.98 & 15.5 & 12.47 & 3.12 \\
\hline $\mathbf{N}_{4} \mathbf{S}_{3}$ & 64.16 & 21.37 & 12.56 & 4.18 \\
\hline $\mathbf{N}_{4} \mathbf{S}_{4}$ & 66.1 & 10.97 & 12.80 & 2.13 \\
\hline Mean (N x S) & 59.02 & 18.33 & 11.81 & 3.67 \\
\hline S.Em \pm & 0.84 & 0.27 & 0.06 & 0.02 \\
\hline CD at $5 \%$ & 2.44 & 0.77 & 0.16 & 0.06 \\
\hline
\end{tabular}


The interaction between nitrogen and plant densities differs significantly for CGR. The maximum CGR was observed in $\mathrm{N}_{4} \mathrm{~S}_{1}$ $\left(13.21 \mathrm{~g} / \mathrm{m}^{2} /\right.$ day) whereas the minimum CGR was recorded in $\mathrm{N}_{2} \mathrm{~S}_{4}$ (3.22 g/day). At 46-60 Days after sowing, the nitrogen level $\mathrm{N}_{4}$ recorded maximum CGR $\left(9.04 \mathrm{~g} / \mathrm{m}^{2} /\right.$ day $)$ and minimum CGR $\left(6.61 \mathrm{~g} / \mathrm{m}^{2} /\right.$ day $)$ was recorded in $\mathrm{N}_{1}$. The plants spaced at $30 \times 20 \mathrm{~cm}\left(\mathrm{~S}_{1}\right)$ recorded the maximum CGR (11.34 g/day) and minimum was recorded in $\mathrm{S}_{4}$ (4.28 $\mathrm{g} / \mathrm{m}^{2} /$ day).

The interaction between nitrogen and plant densities differs significantly for CGR. The higher CGR was observed in treatment combination of $\mathrm{N}_{4} \mathrm{~S}_{1}\left(13.49 \mathrm{~g} / \mathrm{m}^{2} /\right.$ day $)$ and least CGR was recorded in $\mathrm{N}_{1} \mathrm{~S}_{4}$ (3.72 $\mathrm{g} / \mathrm{m}^{2} /$ day). At $61-75$ Days after sowing, the nitrogen level $\mathrm{N}_{4}$ recorded maximum CGR $\left(13.34 \mathrm{~g} / \mathrm{m}^{2} /\right.$ day $)$ and minimum CGR (8.02 $\mathrm{g} / \mathrm{m}^{2} /$ day) was recorded in $\mathrm{N}_{1}$. The plants spaced at $20 \times 10 \mathrm{~cm}\left(\mathrm{~S}_{1}\right)$ recorded the maximum CGR (16.85 $\mathrm{g} / \mathrm{m}^{2} /$ day) while the minimum was recorded in $\mathrm{S}_{4}\left(6.36 \mathrm{~g} / \mathrm{m}^{2} /\right.$ day $)$.

The interactions between nitrogen and plant densities differ significantly for CGR. The maximum CGR were observed in treatment combination of $\mathrm{N}_{4} \mathrm{~S}_{1}\left(20.83 \mathrm{~g} / \mathrm{m}^{2} /\right.$ day and minimum CGR was observed in $\mathrm{N}_{1} \mathrm{~S}_{4}$ (4.71 $\mathrm{g} / \mathrm{m}^{2} /$ day).

The cumulative growth rate was significant at all stages of crop growth. This might be due to higher dose of nitrogen which helps in dry matter accumulation in the plants. Similar trend was observed by Santhosh et al., (2010) in garden cress.

\section{Chlorophyll contents}

The data regarding the Chlorophyll content as influenced by different nitrogen, spacing levels and their interactions are presented in table 4 .

\section{Content of chlorophyll- a}

Among the nitrogen levels, $\mathrm{N}_{4}(80 \mathrm{~kg} \mathrm{~N} / \mathrm{ha})$ recorded significantly higher chlorophyll- a (1.61 $\mathrm{mg} / 100 \mathrm{mg}$ of tissue). While, the minimum chlorophyll-a content $(0.78$ $\mathrm{mg} / 100 \mathrm{mg}$ of tissue) was noticed in the $\mathrm{N}_{1}$ (20 kg N/ha).

Among the spacing levels, the plants spaced at $30 \times 20 \mathrm{~cm}\left(\mathrm{~S}_{4}\right)$ recorded significantly higher chlorophyll- a content $(1.55 \mathrm{mg} / 100 \mathrm{mg}$ of tissue) and minimum chlorophyll- a content $(1.00 \mathrm{mg} / 100 \mathrm{mg}$ of tissue $)$ was noticed in $S_{1}(20 \times 10 \mathrm{~cm})$. The significant variations on in chlorophyll-a content at harvest due to interaction of nitrogen levels and plant densities.

The maximum chlorophyll-a content was observed in treatment combination of $\mathrm{N}_{4} \mathrm{~S}_{4}$ (1.80 mg/100mg of tissue) followed by, $\mathrm{N}_{4} \mathrm{~S}_{2}$ (1.71 mg/100mg of tissue) and $\mathrm{N}_{4} \mathrm{~S}_{3}$ (1.72 $\mathrm{mg} / 100 \mathrm{mg}$ of tissue). While the minimum $(0.14 \mathrm{mg} / 100 \mathrm{mg}$ of tissue) chlorophyll-a content was observed in $\mathrm{N}_{1} \mathrm{~S}_{1}$.

\section{Content of chlorophyll- b}

Among the nitrogen levels, $\mathrm{N}_{4}$ recorded significantly maximum chlorophyll- b (1.38 $\mathrm{mg} / 100 \mathrm{mg}$ of tissue). While the minimum chlorophyll-b $(0.30 \mathrm{mg} / 100 \mathrm{mg}$ of tissue $)$ was noticed in the $\mathrm{N}_{1}(20 \mathrm{~kg} \mathrm{~N} / \mathrm{ha})$. Among the spacing levels, the plants spaced at $30 \times 20$ $\mathrm{cm}\left(\mathrm{S}_{4}\right)$ recorded significantly maximum chlorophyll- b $(0.96 \mathrm{mg} / 100 \mathrm{mg}$ of tissue $)$.

However, the minimum $(0.48 \mathrm{mg} / 100 \mathrm{mg}$ of tissue) chlorophyll- b content was noticed in $\mathrm{S}_{1}$. The significant variations were observed in chlorophyll-b content at harvest due to interaction of nitrogen and spacing levels. The maximum chlorophyll- $b$ content observed in treatment combination of $\mathrm{N}_{4} \mathrm{~S}_{4}$ (1.67 $\mathrm{mg} / 100 \mathrm{mg}$ of tissue) and was followed by 
$\mathrm{N}_{4} \mathrm{~S}_{3}$ (1.55 mg/100mg of tissue). While, the minimum $(0.04 \mathrm{mg} / 100 \mathrm{mg}$ of tissue) chlorophyll-b content observed in $\mathrm{N}_{1} \mathrm{~S}_{1}$.

\section{Total chlorophyll content}

Among the nitrogen levels, $\mathrm{N}_{4}(80 \mathrm{~kg} \mathrm{~N} / \mathrm{ha})$ recorded significantly higher total chlorophyll content $(2.99 \mathrm{mg} / 100 \mathrm{mg}$ of tissue). While, the minimum total chlorophyll content (1.08 $\mathrm{mg} / 100 \mathrm{mg}$ of tissue) was noticed in the $\mathrm{N}_{1}(20$ $\mathrm{kg} \mathrm{N} / \mathrm{ha})$. Among the spacing levels, the plants spaced at $30 \times 20\left(\mathrm{~S}_{4}\right)$ recorded significantly higher total chlorophyll content (2.51 $\mathrm{mg} / 100 \mathrm{mg}$ of tissue). However, the minimum total chlorophyll content $(1.47 \mathrm{mg} / 100 \mathrm{mg}$ of tissue) was noticed in $S_{1}$.

The significant variations were recorded for chlorophyll content at harvest due to interaction of nitrogen levels and plant densities. The maximum total chlorophyll content recorded in the treatment combination of $\mathrm{N}_{4} \mathrm{~S}_{4} \quad(3.48$ $\mathrm{mg} / 100 \mathrm{mg}$ of tissue) and was followed by $\mathrm{N}_{4} \mathrm{~S}_{3}$ (3.26 mg/100mg of tissue). The minimum (0.17 $\mathrm{mg} / 100 \mathrm{mg}$ of tissue) chlorophyll-a content observed in $\mathrm{N}_{1} \mathrm{~S}_{1}$.

The chlorophyll content in leaves differed significantly due to different levels of nitrogen. This might be due to increased nitrogen supply which would retard leaf senescence and improve photosynthate and nitrogen availability for seed biomass. Similar results were noticed by Mitra and Ghildiyal (1988) and Kulsum et al., (2007) in black gram.

\section{Yield parameters}

Observation pertaining to fresh herbage yield per plant as influenced by different levels of nitrogen, spacing and their interactions are presented in table 5. Among the nitrogen levels, application of $\mathrm{N}_{4}$ recorded the maximum total fresh yield per plant $(63.36 \mathrm{~g})$. While the, minimum total fresh yield per plant $(55.23 \mathrm{~g})$ was recorded in $\mathrm{N}_{1}$. Significantly higher fresh yield per plant (60.36 g) was recorded in $S_{4}$ and it was on par with $S_{3}(59.54 \mathrm{~g})$. Whereas, the lowest total fresh yield per plant (57.77 g) was recorded in $S_{1}$. The interaction between nitrogen and spacing levels differ significantly for total fresh yield per plant.

The highest fresh yield per plant was observed in $\mathrm{N}_{4} \mathrm{~S}_{4}(66.10 \mathrm{~g})$ and $\mathrm{N}_{4} \mathrm{~S}_{3}(64.16 \mathrm{~g})$ was found to be next best treatment. While, the minimum total fresh yield per plant was recorded in $\mathrm{N}_{1} \mathrm{~S}_{1}$ $(54.02 \mathrm{~g})$. The maximum total fresh yield per hectare $\left(19.61 \mathrm{t}\right.$ ) was noticed in $\mathrm{N}_{4}$. While, the minimum fresh yield per hectare $(17.15 \mathrm{t}$ ) was recorded in $N_{1}$. The $S_{1}$ recorded significantly higher total fresh yield per hectare $(28.88 \mathrm{t})$. Whereas, the lowest total fresh yield per hectare (10.02) was recorded in $S_{4}$.

The nitrogen level $\mathrm{N}_{4}$ exhibited higher total dry yield per plant (12.55 g) while, the least total dry yield per plant (11.00 g) was observed in $\mathrm{N}_{1}$. Among the different spacing levels, $\mathrm{S}_{4}$ recorded significantly higher dry yield per plant $(12.04 \mathrm{~g})$. Whereas, the lowest dry yield per plant $(11.60 \mathrm{~g})$ was noticed in $\mathrm{S}_{1}$. The dry yield per plant varied significantly due to interaction of nitrogen and spacing levels. The maximum $(12.80 \mathrm{~g})$ and minimum $(10.74 \mathrm{~g})$ dry yield per plant was reported in $\mathrm{N}_{4} \mathrm{~S}_{4}$ and $\mathrm{N}_{1} \mathrm{~S}_{1}$ treatment combination, respectively.

The maximum total dry yield per hectare (3.90 t) was recorded in $\mathrm{N}_{4}(80 \mathrm{~kg} \mathrm{~N} / \mathrm{ha})$ and minimum total dry yield per hectare $(3.42 \mathrm{t})$ observed in $\mathrm{N}_{1}$. The closer spacing of $20 \times 10$ $\mathrm{cm}\left(\mathrm{S}_{1}\right)$ recorded significantly higher total dry yield per hectare $(5.80 \mathrm{t})$. Whereas, the lowest total dry yield per hectare $(2.0 \mathrm{t})$ was noticed in $\mathrm{S}_{4}$. The interaction between nitrogen and spacing levels differed significantly for total dry yield per hectare. The maximum total dry yield per hectare was observed in $\mathrm{N}_{4} \mathrm{~S}_{1}(6.19 \mathrm{t})$ whereas, the minimum total dry yield per hectare was recorded in $\mathrm{N}_{1} \mathrm{~S}_{4}(1.87 \mathrm{t})$.

The fresh and dry yield per plant, per plot and per hectare differed significantly due to different levels of nitrogen. The increase in yield might be attributed to the fact that under increasing nitrogen levels, there would be 
luxuriant growth of the plant which is evident from vegetative growth, which led to the production of more fresh and dry herbage yield. These results are conformity with the findings of Bhaskar (1995) in patchouli, Balyan and Sobti (1990) in Ocimum gratissimum, Meena et al., (2006) in fenugreek and Datta et al., (2005) in fenugreek.

From the present investigation, it can be concluded that the Kasuri methi is beneficial for obtaining the maximum physiological and higher yield parameters, under the northern dry zone of Karnataka.

\section{References}

Anonymous, 2004. Trigonella corniculata Linn., Wealth of India, Raw materials, CSIR, New Delhi, 5: 252-258.

Balyan, S.S., and Sobti, S.N., 1990. Effect of inter and intra row spacing on growth, yield and eugenol content in Ocimum gratissimum L. (Clocimum). Indian Perfumer, 34: 217-224.

Bhaskar, S., 1995. Growth, herbage and oil yields of patchouli (Pogostemon patchouli) as influenced by cultivars and nitrogen fertilization. Indian Perfumer, 39: 35-38.

Datta, S., Kalam and Chatterjee, R., 2005. Effect of different levels of nitrogen and leaf cutting on growth and seed yield of fenugreek. Ind. J. Agri. Sci., 75(9): 580581.

Kattimani, K.N., 1999. Effect of planting material, spacing, nitrogen, phosphorous and harvest intervals on growth, biomass, nutrient uptake, oil yield and quality of
Japanesemint (Mentha arvensis L.) Ph.D. Thesis, Acharya N.G. Ranga. Agric. Univ. Hyderabad.

Kulsum, M.U., Baque, M. A., and Karim, M. A., 2007. Effects of Different Nitrogen levels on the leaf chlorophyll content nutrient concentration and nutrient uptake pattern of black gram. Pak. J. Biol. Sci., 10: 250-254.

Meena, S.S., Sen, N. L. and Malhotra, S. K., 2006. Influence of nitrogen on growth and yield of Coriander (Coriandrum sativum L.). J. Spices Arom. Crops, 15 (2): 88-92.

Mehta, R.S., Patel, B.S., Meena, S. S., and Meena, R. S., 2010. Influence of growth characters and yield of fenugreek (Trigonella foenum-graecum). J. Med. Arom. Plant Sci., 19(2): 23-28.

Mitra, S., and Ghildiyal, M.C., 1988. Photosynthesis and assimilate partitioning in mungbean in response to source sink alteration. J. Agron. Crop. Sci., 160: 303308.

Panse, V.G., and Sukhatme, P.V., 1967. Stastical Methods for Agricultural Workers, Indian council of Agricultural Research, New Delhi.

Patidar, M., Balaram, B., Singh, M. P. and Singh, G., 2004. Influence of nitrogen on growth and yield of Cumin (Cuminum cyminum L.). J. Spices Arom. Crops, 13 (2): 126-128.

Santosh, C., Keshwa, G. L. and Yadav, L. R., 2010. Effect of row spacings and nitrogen levels on productivity of garden cress (Lepidium sativum). Ind. J. Agric. Sci., 80 (8): $752-754$.

\section{How to cite this article:}

Anupama, G., L.N. Hegde, N.K. Hegde, V. Devappa, A.B. Mastiholi and Sandhyarani Nishani. 2017. Effect of Nitrogen and Spacing Levels on Physiological and Yield Parameters of Kasuri Methi (Trigonella corniculata L.) var. Pusa Kasuri. Int.J.Curr.Microbiol.App.Sci. 6(9): 723-733. doi: https://doi.org/10.20546/ijcmas.2017.609.089 